\title{
Guías latinoamericanas para el manejo de la emesis en oncología, hematooncología y radioterapia*
}

\author{
Latin-American guidelines for the management of emesis in oncology, \\ haemato-oncology, and radiotherapy*
} \author{
Andrea Ponce de León-Herrera ${ }^{10}$, Ivo Rodríguez ${ }^{11}$, José Federico Rojas-Montero ${ }^{12}$, \\ Juan Fernando Miguel Suazo-Casanova ${ }^{13}$, María Alejandra Torres ${ }^{14}$, Carlos Vargas ${ }^{15,16}$ \\ "Instituto Nacional de Cancerología de México, presidente de la Academia Mexicana de Cirugía (México D.F., México). \\ ${ }^{2}$ Clínica de Oncología Médica, Hospital Brigadeiro (São Paulo, Brasil). \\ ${ }^{3}$ Servicio de Oncología Médica, CMN 20 de Noviembre, ISSSTE (México D.F., México). \\ ${ }^{4}$ Hospital General de Agudos Santojanni (Buenos Aires, Argentina). \\ ${ }^{5}$ Clínica Latinoamericana, Solca (Cuenca, Ecuador). \\ ${ }^{6}$ Hospital de Pediatría CMN Siglo XXI (México D.F., México). \\ ${ }^{7}$ Fundación Arturo López Pérez (Santiago de Chile, Chile). \\ ${ }^{8}$ Sociedad de Oncología y Hematología del Cesar (Valledupar, Colombia). \\ Instituto Salvadoreño, Seguro Social (San Salvador, El Salvador). \\ ${ }^{10}$ Servicio de Oncología Pediátrica, Hospital de Pediatría CMN Siglo XXI (México D.F., México). \\ "Servicio de Radioterapia y Radiocirugia, Hospital de Clinicas Caracas (Caracas, Venezuela). \\ "Servicio de Oncologla, Hospital San Juan de Dios (San Jose, Costa Rica. \\ is Departamento de Oncologia, Oncosalud-AUNA (Lima, Perú). \\ Santa Fe de Bogotá (Bogotá Colombia). \\ ${ }^{16}$ Fundación para la Investigación Clínica y Molecular Aplicada del Cáncer (FICMAC) (Bogotá, Colombia).
}

- Francisco Javier Ochoa-Carrillo1, Ricardo Caponero², María Guadalupe Cervantes-Sánchez³, Óscar Daniel Duarte ${ }^{4}$ María Isabel León ${ }^{5}$, Enrique López-Aguilar ${ }^{6}$, Mauricio Mahave, Raimundo Manneh ${ }^{8}$, Álvaro Menéndez-Leal9

\section{Resumen}

El tratamiento del paciente oncológico es complejo debido a que la naturaleza de la patología requiere un manejo sistémico. Las distintas terapias que buscan restablecer la salud de los pacientes, o al menos procurarles calidad de vida, deben modificarse constantemente, porque sus efectos adversos a menudo provocan que los pacientes interrumpan su tratamiento o lo abandonen por completo. Tal es el caso de la náusea y el vómito inducidos por radioterapia y quimioterapia, que provocan mayor malestar a los pacientes y que, desde hace más de dos décadas, han tratado de ser controlados con antieméticos cada vez más efectivos. Desde hace tiempo, diversas instituciones oncológicas internacionales han publicado guías para homologar los criterios del manejo de la náusea y vómito inducidos por quimioterapia; sin embargo, hasta el momento, se ha carecido de guías y lineamientos específicos adaptados para Latinoamérica, región donde existen circunstancias particulares referentes a la disposición de fármacos y al acceso a recursos. Ante ese panorama, un grupo de especialistas oncólogos, hematólogos y oncólogos pediátricos de Latinoamérica se reunió con el propósito de consensuar el manejo general y las recomendaciones específicas sobre el uso de los fármacos antieméticos, solos o en combinación, y conformar las guías del manejo de la emesis, de acuerdo con las particularidades del contexto latinoamericano. Los expertos contestaron un cuestionario Delphi que contenía los conceptos fundamentales del tratamiento antiemético. Los calificaron mediante parámetros de acuerdo y desacuerdo. Posteriormente, se reunieron para llegar a un consenso en los que requerían discusión y revisaron los reportes más relevantes de las publicaciones internacionales (incluidas las guías de MASCC, NCCN y ASCO). Finalmente, se redactaron las guías latinoamericanas para el control de la emesis.

Palabras clave: Quimioterapia, radioterapia, efectos adversos, emesis, vómito, náusea, antineoplásicos, antieméticos.

\begin{abstract}
The treatment for the oncological patient is complex because the pathology in itself requires a systemic management. The different therapies -which search for restoring the health of the patients, or at least improving their quality of life- constantly must be modified because their adverse effects usually make the patients to interrupt the treatment or abandon it altogether. Such is the case of nausea and vomiting induced by radiotherapy and chemotherapy, which cause troublesome
\end{abstract}

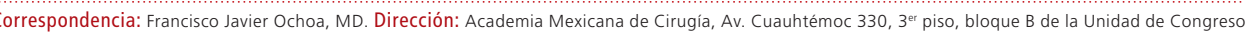
del Centro Médico Nacional Siglo XXI, Col. Doctores, C.P. 06720, México D.F., México. Teléfonos: (+54) 5588-0458/5761-2581 / 5568-5013 / $4596-0294$. Correo electrónico: ochoacarrillo@prodigy.net.mx

Fecha de recepción: 22 de enero del 2013. Fecha de aprobación: $1^{\circ}$ de marzo del 2013.

Declaración de conflictos de interés: ninguno.

*Este artículo se reproduce sin modificaciones por autorización del Comité Editorial de la Gaceta Mexicana de Oncología (GAMO), órgano oficial de divulgación científica de la Sociedad Mexicana de Oncología (SMeO). 
ailments to the patients and that have been treated with more effective antiemetics for over two decades. Long time ago, several international oncological institutions have published guidelines to homogenize the management criteria of the nausea and vomiting induced by chemotherapy. Nevertheless, there has been a lack of specific and adaptive guidelines for LatinAmerica, region where there are particular circumstances related to drugs availability and access to resources. Taking all this into account, a group of oncologists, haematologists, and paediatric oncologists from Latin-America gathered with the purpose of agreeing about the general management and specific recommendations of the antiemetic drugs administration alone or in combination; as well as to establish the management of emesis guidelines according to the Latin-American context. The experts answered a Delphi questionnaire which contained the basic concepts of the antiemetic treatment. They graded them with the criteria agree and disagree. Later on, they assembled again to discuss the controversial concepts, reviewed the most outstanding literature reports (including the MASCC, NCCN, and ASCO guidelines), and finally, they draw up the Latin-American guides for the control of emesis.

Key words: Chemotherapy, radiotherapy, adverse effects, emesis, vomiting, nausea, antineoplastic, antiemetics.

\section{Introducción}

Pese a que la quimioterapia y la radioterapia -solas o combinadas- son opciones terapéuticas en el manejo del paciente oncológico, la náusea y el vómito que provocan ponen en riesgo la salud de los pacientes, porque disminuyen la adhesión al tratamiento, alteran el equilibrio hidroelectrolítico e, incluso, pueden producir desnutrición ${ }^{1-4}$.

Ante este panorama, los autores de este documento colaboraron con la Sociedad Mexicana de Oncología, la Asociación Brasileña de Cuidados Paliativos, la Sociedad Ecuatoriana de Oncología y otras sociedades oncológicas latinoamericanas, con el propósito de conformar un conjunto de lineamientos para el manejo de la emesis, en concordancia con las particularidades del contexto latinoamericano. De acuerdo con Chambers (tabla 1) el vómito y la náusea pueden clasificarse en cinco grados (1 a 5), donde el 1 es el mínimo y el 5 el más grave. Así mismo, el vómito y la náusea pueden presentarse de modo independiente, pero su patogénesis es la misma; es decir, se originan en un estímulo recibido por el centro del vómito (CV) en el bulbo raquídeo. Los estímulos que llegan al CV son mediados principalmente por los nervios simpáticos y vagales, después de la activación de al menos una de las siguientes cuatro zonas ${ }^{4}$ :

1. Zona activadora quimiorreceptora, en la que se encuentran los receptores de dopamina y serotonina.
2. Zona vestibular, la cual origina el vómito cuando se estimulan los receptores muscarínicos-colinérgicos de histamina $(\mathrm{H} 1)$.

3. Zona gastrointestinal y biliar, estimulada por distensión e irritación peritoneal y de la mucosa gastrointestinal.

4. La corteza cerebral, donde tiene lugar la náusea y el vómito anticipatorios.

Ambas alteraciones han podido ser controladas durante dos décadas, pero aún no se han logrado prevenir los síntomas del todo, debido a que son resultado de un complejo conjunto de factores ${ }^{3}$. Primero, se pensaba que el vómito y la náusea eran provocados por la reacción concentrada en el $\mathrm{CV}^{6}$. A ello se sumó el descubrimiento de vías de señalización neurológica-que parten desde el sistema límbico y el cerebro- involucradas en el mecanismo de la náusea y el vómito después de recibir un estímulo psicológico, como el estrés, o alguna emoción, como la ansiedad ${ }^{7,8}$. Luego, se afirmó que el causante del reflejo de náusea-vómito es un conjunto de neuronas y receptores (5-HT3 y neurocinina 1 ) que se encuentran en la zona gatillo y el $\mathrm{CV}^{8}$.

El 5-HT3 se encarga sobre todo de los vómitos agudos al ser estimulado por la serotonina, que, en mayor medida, se libera en las células enterocromafines del duodeno; la neurocinina (NK-1), en cambio, provoca vómitos tardíos por la liberación y agonismo de la

Tabla 1. Graduación del vómito y nausea

\begin{tabular}{|c|c|c|c|c|c|}
\hline Condición & Grado 1 & Grado 2 & Grado 3 & Grado 4 & Grado 5 \\
\hline Vómito & $\begin{array}{l}\text { Pérdida de apetito } \\
\text { sin alteración } \\
\text { de los hábitos } \\
\text { de alimentación }\end{array}$ & $\begin{array}{l}\text { Disminución de la ingesta } \\
\text { sin pérdida significativa } \\
\text { de peso, deshidratación, } \\
\text { desnutrición o uso de } \\
\text { líquidos IV por menos de } \\
24 \text { horas }\end{array}$ & $\begin{array}{c}\text { Ingesta inadecuada } \\
\text { de líquidos o calórica, } \\
\text { con requerimiento de } \\
\text { rehidratación por vía IV, } \\
\text { alimentación parenteral o } \\
\text { TPN por un período mayor } \\
\text { a } 24 \text { horas }\end{array}$ & $\begin{array}{l}\text { Consecuencias que ponen } \\
\text { en riesgo la vida }\end{array}$ & Muerte \\
\hline Náusea & Un episodio en 24 horas & $\begin{array}{l}2-5 \text { episodios en } 24 \text { horas y } \\
\text { requerimiento de líquidos } \\
\text { IV por menos de } 24 \text { horas }\end{array}$ & $\begin{array}{l}>6 \text { episodios en } 24 \text { horas o } \\
\text { requerimiento de TPN por } \\
\text { más de } 24 \text { horas }\end{array}$ & $\begin{array}{l}\text { Consecuencias que ponen } \\
\text { en riesgo la vida }\end{array}$ & Muerte \\
\hline
\end{tabular}


sustancia P en el sistema nervioso central. Sin duda, todo ese conocimiento ha sido el punto de partida para poder cumplir los principales objetivos del tratamiento antiemético: evitar el vómito y la náusea inducidos por quimioterapia (chemotherapy-induced nausea and vomiting, CINV) y radioterapia (radiation-induced nausea and vo-miting, RINV), preservar la calidad de vida de los pacientes bajo tratamiento oncológico y disminuir los costos del sistema de salud.

\section{Clasificación de la CINV}

La CINV se divide en: 1) aguda, 2) tardía y 3) anticipatoria. La aguda se presenta entre las 0 y 24 horas posteriores a la quimioterapia; la tardía aparece de 24 a 120 horas después de haber recibido el agente antineoplásico; la anticipatoria ocurre antes de la administración de la quimioterapia, a causa de algún sabor, olor, imagen, pensamiento o ansiedad, sobre todo en los pacientes que ya han recibido varios ciclos de quimioterapia ${ }^{1,9,10}$.

\section{Emetogenicidad de los fármacos antineoplásicos}

Contar con una clasificación del grado de emetogenicidad de los antineoplásicos es de gran utilidad, ya que ello facilita la elección del antiemético más adecuado para los pacientes. En general, a los antineoplásicos se los clasifica como de riesgo emetogénico elevado

Tabla 2. Clasificación del riesgo emetogénico de la quimioterapia

\begin{tabular}{|c|c|}
\hline Riesgo & Fármaco \\
\hline Alto $(>90 \%)$ & $\begin{array}{l}\text { Intravenoso } \\
\text { Cisplatino ( } 50 \mathrm{mg} / \mathrm{m}^{2} \text { o más) } \\
\text { Oral } \\
\text { Temozolamida a dosis altas ( } 150 \mathrm{mg} / \mathrm{m}^{2} / \text { día o más) } \\
\text { Mecloretamina } \\
\text { Estreptozotocina } \\
\text { Dacarbazina } \\
\text { Ciclofosfamida }>1.500 \mathrm{mg} / \mathrm{m}^{2} \\
\text { Cominaciones AC (doxorrubicina/ciclofosfamida) o EC } \\
\text { (epirrubicina/ciclofosfamida) }\end{array}$ \\
\hline Moderado (30-90\%) & $\begin{array}{l}\text { Procarbazina } \\
\text { Intravenoso } \\
\text { Oxaliplatino } \\
\text { Citarabina }>1.000 \mathrm{mg} / \mathrm{m}^{2} \\
\text { Ciclofosfamida }<1.500 \mathrm{mg} / \mathrm{m}^{2} \\
\text { Doxorrubicina } \\
\text { Epirrubicina } \\
\text { Daunorrubicina } \\
\text { Idarrubicina } \\
\text { Irinotecan } \\
\text { Vinblastina } \\
\text { Oral } \\
\text { Ciclofosfamida } \\
\text { Etoposido } \\
\text { Temozolamida a dosis bajas }\left(75 \mathrm{mg} / \mathrm{m}^{2} / \mathrm{dia}\right) \\
\text { Imatinib }\end{array}$ \\
\hline
\end{tabular}

(90\%), moderado (30-90\%), bajo (10-30\%) y mínimo $(<10 \%)$ (tabla 2$)^{9,10}$.

Más de dos décadas de investigaciones y artículos publicados respecto al tema han encontrado que la terapia antiemética para los pacientes tratados con agentes antineoplásicos puede prevenir del 70 al 80\% de CINV ${ }^{11}$.

Durante el decenio de 1980, el estándar de oro para controlar la emesis inducida por quimioterapia eran las dosis altas de metoclopramida. Una década más tarde se administraban antagonistas de serotonina (5-HT3) + corticosteroides, combinación que resultó efectiva y poco tóxica'2.

\section{Factores de riesgo}

Los factores de riesgo de la CINV más comunes se clasifican en dos:

Relacionados con el tratamiento:

- Tipo de quimioterapia

- Dosis de agentes antineoplásicos

- Momento de la administración

- Vía de administración

Relacionados con los pacientes $1,9,10$ :

- Género femenino

- Paciente joven (menor de 50 años)

- Emesis durante el embarazo

\begin{tabular}{|c|c|}
\hline Riesgo & Fármaco \\
\hline Bajo (10-30\%) & $\begin{array}{l}\text { Intravenoso } \\
\text { Topotecan } \\
\text { Gemcitabina } \\
\text { Doxorrubicina liposomal } \\
\text { Mitoxantrona } \\
\text { Docetaxel } \\
\text { Paclitaxel } \\
\text { Etopósido } \\
\text { Pemetrexed } \\
\text { Metotrexate } \\
\text { Mitomicina } \\
\text { 5-Fluoruracilo } \\
\text { Citarabina }<100 \mathrm{mg} / \mathrm{m}^{2} \\
\text { Bortezomib } \\
\text { Trastuzumab } \\
\text { Oral } \\
\text { Capecitabina } \\
\text { Fludarabina } \\
\text { Topotecan }\end{array}$ \\
\hline Mínimo (< 10\%) & $\begin{array}{l}\text { Intravenoso } \\
\text { Bleomicina } \\
\text { Busulfan (dosis bajas) } \\
\text { Cladibrina } \\
\text { Fludarabina } \\
\text { Vincristina } \\
\text { Vinblastin } \\
\text { Vincristina } \\
\text { Bevacizumab } \\
\text { Cetuximab }\end{array}$ \\
\hline
\end{tabular}


- Antecedentes de CINV

- Mareo durante algún movimiento

- Presencia de algún tumor

- Ansiedad o labilidad emocional

- Reacciones adversas por interacción de fármacos

\section{Recomendaciones generales}

1. A los pacientes que reciben quimioterapia y radioterapia se les deben suministrar antieméticos de acuerdo con la emetogenicidad de la quimioterapia, a menos que el riesgo emético de la radioterapia sea mayor (en abdomen superior) (tabla 3) ${ }^{13}$.

2. Los pacientes que reciben quimioterapia combinada también deben ser tratados con antieméticos apropiados para los agentes antineoplásicos que tienen mayor riesgo emético. Las combinaciones de antraciclinas + ciclofosfamida son altamente emetogénicas ${ }^{13}$.

3. Es recomendable administrar antieméticos según la clasificación del riesgo de emetogenicidad de la quimioterapia. Los pacientes deben recibirlos cada día de la quimioterapia y dos días después de ella. Los pacientes a los que se les brinda regímenes de cisplatino durante cinco días deben ser tratados con antagonistas de 5-HT3 + dexametasona + aprepipant ${ }^{13}$.

4. Los fármacos recomendados para prevención de emesis en quimioterapia IV de alto riesgo emetogénico son granisetrón IV (1 mg máximo $\left.{ }^{11}\right)$, u ondasetrón (16 mg), o palonosetrón (0,25 mg IV) + dexametasona (12 mg IV más 8 mg VO, días 2-4) + aprepitant (125 mg, día $1+80$ mg VO, días 2 y 3), con lorazepam (0,5-1 mg VO, días 1-4) + bloqueador $\mathrm{H} 2 \mathrm{o}$ inhibidor de la bomba de protones, ambos a criterio médico ${ }^{13}$. Así mismo, se sugiere el fosaprepitant (150 mg IV), en dosis única el primer día. Cuando se administra VO, las dosis son 125 mg el día 1 y $80 \mathrm{mg}$ los días 2 y $3^{15}$. Con todo, la combinación fosaprepitant + palonosetrón + dexametasona es en verdad efectiva.

5. Cuando los pacientes reciben agentes con emetogenicidad mínima, no es necesaria la administración de antieméticos ${ }^{13}$.

6. En casos de náusea y vómito anticipatorios, se sugiere la administración de antieméticos activos para prevenir emesis aguda o tardía. Estos pueden administrarse en quimioterapia inicial, más que evaluar la respuesta emética de los pacientes con un tratamiento menos efectivo. Si sobreviene un episodio de emesis anticipatoria, se sugiere una terapia de conducta, acompañada de la desensibilización sistemática ${ }^{13}$. No obstante, es preciso recordar que el mejor tratamiento antiemético es la prevención.

7. Los pacientes jóvenes que han experimentado con frecuencia náusea y vómito intensos después de los primeros tratamientos con quimioterapia presentan un riesgo alto de desarrollar vómito y náusea anticipatorios ${ }^{16}$.

8. El mejor tratamiento para la emesis anticipatoria es el control de las emesis aguda y tardía ${ }^{16}$.

9. La náusea y el vómito anticipatorios son difíciles de controlar mediante fármacos, de ahí que se recurra a terapias de conducta, particularmente desensibilización sistemática ${ }^{17}$. Cuando existe un bloqueo completo (triple esquema: aprepitant + dexametasona + palonosetrón), la náusea y el vómito disminuyen. Si bien el mecanismo no es muy conocido, parece que, al bloquear los de tipo agudo y tardío, la dopamina no cumple su papel de memoria-recompensa y no quedan registrados.

Tabla 3. Grado de emetogenicidad de la radioterapia

\begin{tabular}{|c|c|c|c|c|}
\hline Nivel de riesgo & Área irradiada & $\begin{array}{c}\text { Guías manejo } \\
\text { de náusea y emesis }\end{array}$ & Nivel de consenso MASCC & $\begin{array}{l}\text { Nivel de evidencia/grado } \\
\text { de recomendación ESMO }\end{array}$ \\
\hline Alto (> 90\%) & $\begin{array}{l}\text { Irradiación corporal total, } \\
\text { irradiación ganglionar total }\end{array}$ & $\begin{array}{l}\text { Profilaxis con antagosnistas de } \\
\text { 5HT3 + dexametasona }\end{array}$ & $\begin{array}{l}\text { Alta recomendación } \\
\text { Adición de dexametasona (grupo } \\
\text { moderada/alta emetogenicidad) }\end{array}$ & $\begin{array}{l}\text { IIB (para la adición de } \\
\text { dexametasona IIC) }\end{array}$ \\
\hline $\begin{array}{l}\text { Moderado } \\
(60-90 \%)\end{array}$ & $\begin{array}{l}\text { Irradiación al abdomen superior, } \\
\text { hemicorporal o del hemicuerpo } \\
\text { superior }\end{array}$ & $\begin{array}{l}\text { Rescate con antagonistas de las } \\
\text { receptores de la dopamina }\end{array}$ & $\begin{array}{l}\text { Alta recomendación } \\
\text { Adición de dexametasona (grupo } \\
\text { moderada/alta emetogenicidad) }\end{array}$ & $\begin{array}{l}\text { IIA (para la adición de } \\
\text { dexametasona IIB) }\end{array}$ \\
\hline $\begin{array}{l}\text { Bajo } \\
(30-60 \%)\end{array}$ & $\begin{array}{l}\text { Irradiación craneal o craneoespinal, } \\
\text { de cabeza y cuello, de la región } \\
\text { baja del tórax o de la pelvis }\end{array}$ & $\begin{array}{l}\text { Rescate con antagonistas de las } \\
\text { receptores de la dopamina }\end{array}$ & $\begin{array}{l}\text { Moderada/alta recomendación } \\
\text { Adición de dexametasona (para } \\
\text { rescate baja/alta recomendación) }\end{array}$ & IIB (para rescate N/C) \\
\hline $\begin{array}{l}\text { Mínimo } \\
(<30 \%)\end{array}$ & Extremidades, tórax & $\begin{array}{l}\text { Antagonistas de } 5 \mathrm{HT} 3+ \\
\text { dexametasona }\end{array}$ & Bajo/alto & N/D \\
\hline
\end{tabular}

Fuente: adaptado de ficha 2010 
10. En caso de no tener respuesta con el triple esquema (aprepitant + dexametasona + palonosetrón), las principales técnicas conductuales que podrían controlar la náusea y el vómito anticipatorios son hipnosis, biorretroalimentación, yoga, entrenamiento de relajación muscular progresiva, desensibilización sistemática ${ }^{16}$.

11. Una dosis baja de lorazepam (0,5 a 2 mg/día), complementaria a un programa de apoyo psicológico que incluye entrenamiento de relajación muscular progresiva, es efectiva y también controla el insomnio ${ }^{16}$

12. El lorazepam y la difenhidramina son fármacos complementarios a los antieméticos, pero no se recomiendan como monoterapia ${ }^{13}$.

13. En los casos de vómito y náusea inducidos por radioterapia de alta emetogenicidad, se aconseja que todos los pacientes reciban antagonistas de 5-HT3 antes de cada fracción, al menos durante 24 horas posteriores a la radioterapia. A los pacientes también se les debe dar dexametasona por cinco días durante las fracciones 1 a $5^{13}$.

14. Los 5-HT3, NK-1-RA y los corticosteroides son los antieméticos más importantes ${ }^{13}$.

15. A pesar de que la mayoría de los pacientes tratados con antieméticos logra una protección completa con 5-HT3, NK-1-RA y corticosteroides, un número considerable sigue cursando con CINV ${ }^{13}$.

16. Los antagonistas del receptor de serotonina (5-HT3) y los antagonistas del receptor de neurocinina 1 (NK1-RA) son efectivos como terapia contra CINV ${ }^{10,18}$.

17. El concepto de profilaxis antiemética de un día requiere mayor investigación ${ }^{13}$.

18. Los pacientes en tratamiento con agentes emetogénicos bajos deben recibir una sola dosis de $8 \mathrm{mg}$ de dexametasona antes de la quimioterapia ${ }^{13}$. En su defecto, es posible utilizar un bloqueador del receptor de $5-\mathrm{HT}^{14,19}$.

\section{Recomendaciones para el uso de antagonistas del receptor de 5-HT3, corticosteroides, benzodiacepinas, antagonistas del receptor de neurocinina 1 , terapia combinada y canabinoides}

1. A pesar de que la profilaxis sea óptima, los pacientes pueden experimentar náusea y vómito, antes de concluir que se trata de un fracaso terapéutico. Los especialistas deben evaluar los factores de riesgo individuales (opioides, hipercalcemia, metástasis al SNC, obstrucción intestinal, entre otros). Una vez excluidos estos factores, es posible complementar con lorazepam y metoclopramida, e incrementar la dosis de esteroides o cambiar el setrón ${ }^{14,20}$.

2. Los antagonistas del receptor de serotonina o 5 -HT3 son eficaces y seguros en la emesis aguda ${ }^{10}$.

3. Dado que el palonosetrón se une con el receptor de 5-HT3 (100 veces más que ondansetrón, granisetrón y dolasetrón, y una vida media de 40 horas'), proporciona mayor control de la náusea y el vómito, comparado con los antagonistas de 5-HT3, en especial durante las 24 a 120 horas posteriores a la quimioterapia ${ }^{13}$.

4. El palonosetrón, al igual que los otros antagonistas de los receptores de 5-HT3, no se recomienda como monoterapia ${ }^{21}$. Debe ser usado con dexametasona (doble esquema) en riesgo moderado. En caso de riesgo bajo de emetogenicidad, puede ser una opción.

5. Los antagonistas inhibidores de serotonina han sido los antieméticos más empleados en el manejo de CINV ${ }^{13}$.

6. En la actualidad, se encuentran disponibles cinco antagonistas del receptor de serotonina o 5-HT3: ondansetrón, granisetrón, tropisetrón, dolasetrón y palonosetrón ${ }^{13}$.

7. En el manejo de náusea retardada (por quimioterapia moderadamente emetogénica), los antagonistas de 5-HT3 son una terapia alternativa a la dexametasona ${ }^{10}$.

8. En la terapia moderadamente emetogénica, el palonosetrón es la primera elección. En caso de no contar con palonosetrón, se puede administrar dolasetrón (solo vía oral), granisetrón u ondasetrón ${ }^{13,14}$.

9. Para los pacientes que reciben radioterapia de emetogenicidad moderada, se recomiendan antagonistas de 5-HT3 antes de cada fracción durante todo el tiempo que se administre la quimioterapia ${ }^{13}$.

10. Cuando los pacientes reciben radioterapia de baja emetogenicidad, se aconseja la administración de antagonistas de serotonina 5-HT3 solo como terapia de rescate. Se sugiere que los pacientes que presentan náusea y vómito inducida por 
radiación -mientras reciben terapia de rescatecontinúen con tratamiento profiláctico hasta que se complete la radioterapia ${ }^{13}$.

11. En caso de intolerancia a los inhibidores de 5-HT3, se puede administrar metoclopramida ${ }^{10}$.

12. Se recomiendan dexametasona y antagonistas de 5-HT3 para pacientes que reciben altas dosis de quimioterapia por trasplante de médula ósea y células progenitoras de sangre periférica ${ }^{13}$.

13. Los pacientes pediátricos que reciben quimioterapia de emetogenicidad moderada o alta deben ser tratados con antagonistas 5-HT3 a una dosis más alta basada en el peso corporal13.

14. El palonosetrón a dosis de 0,25 mg, independientemente del peso, en combinación con dexametasona, es una opción segura y eficaz ${ }^{22}$. Recomendación A1.

15. La adición de dexametasona ( 4 a $8 \mathrm{mg} / \mathrm{m}^{2}$ ) al antagonista 5-HT3 de elección duplica la posibilidad de un control completo de la náusea y vómito en pacientes pediátricos con quimioterapia altamente emetogénica ${ }^{23}$.

16. Los canabinoides son probablemente eficaces, pero producen altos niveles de efectos secundarios en pacientes pediátricos, por lo que no se recomienda su uso ${ }^{23}$.

17. El granisetrón puede ser más eficaz a mayor dosis $(20 \mu \mathrm{g} / \mathrm{kg})$ para el control de pacientes pediátricos que reciben quimioterapia emetogénica moderada ${ }^{23}$.

18. El uso de aprepitant en adolescentes que reciben quimioterapia de alta emetogenicidad es bien tolerado. El régimen de dosificación para adultos es apropiado para adolescentes ${ }^{24}$.

19. En casos de radiación de riesgo moderado, se aconseja un antagonista de serotonina 5-HT3 antes de cada fracción; administrar dexametasona durante cinco días es opcional en la radiación que incluye la región superior del abdomen ${ }^{13}$.

20. Se debe considerar el uso de un esteroide en la terapia moderadamente emetogénica ${ }^{10}$.

21. Se debe considerar la administración de dexametasona durante las fracciones 1 a $5^{13}$.

22. En terapia moderadamente emetogénica, la dosis de dexametasona baja es de 20 a 12 mg; y la administración de lorazepam debe ser opcional en todos los niveles de emetogenicidad ${ }^{13}$.
23. Aunque se desconoce el mecanismo de acción de los esteroides, y no están aprobados como agentes antieméticos, sí se utilizan en combinación con otros agentes antieméticos, pues estos aumentan su efecto terapéutico en la prevención de náusea y vómito inducidos por quimioterapia ${ }^{10}$. La dexametasona es el esteroide más usado (aunque también se podría utilizar metilprednisolona o prednisona en caso de no contar con dexametasona) $)^{10,15,25}$. Se recomienda la administración de $20 \mathrm{mg}$ de dexametasona (12 mg cuando se prescribe aprepitant) en pacientes con riesgo alto, una dosis de $12 \mathrm{mg}$ cuando la quimioterapia es de riesgo moderado y de $8 \mathrm{mg}$ en riesgo bajo $1,10,26,27$.

24. Usar esteroides durante un plazo corto (máximo cinco días) no provoca reacciones adversas importantes, a menos de que tenga una contraindicación relativa como hiperglucemia o hipertensión no controladas $^{10}$.

25. No se recomienda la combinación metoclopramida + esteroides para tratar la prevención de náusea y vómito inducidos por quimioterapia ${ }^{10}$. Recomendación A1.

26. El aprepitant duplica la AUC (área bajo la curva) de la dexametasona'.

27. El aprepitant se combina con dexametasona y palonosetrón para el control de la emesis ${ }^{13}$.

28. Los antagonistas del receptor de neurocinina (aprepitant, fosaprepitant, casopitant, netupitant y rolapitant) inhiben el receptor NK-1 en los sistemas nerviosos central y periférico ${ }^{17}$.

29. El triple esquema aprepitant $+5-\mathrm{HT} 3$ (ondansetrón o palonosetrón) + dexametasona ha demostrado reducir significativamente la emesis aguda y tardía en pacientes que reciben quimioterapias alta y moderadamente eméticas, en comparación con el esquema existente de 5-HT3 + dexametasona para control de emesis aguda y tardía',28,29. Recomendación B-1.

30. Fosaprepipant IV (1 día) y aprepipant oral son equivalentes en respuesta completa y control de emesis y náusea ${ }^{13}$. En dosis de 150 mg, el fosaprepitant sería más efectivo que el aprepitant por su efectividad, potencia antiemética, mayor vida media y cantidad de dosis. El fosaprepitant solo requiere 150 mg IV día 1, con disminución de 
la dosis de dexametasona, contra el aprepitant, que precisa tres dosis de 125,80 y $80 \mathrm{mg}$ con dexametasona en dosis total15,30,31. Recomendación A-1.

31. Algunos estudios sugieren que la gabapentina puede ser efectiva en la profilaxis de náusea y vómito inducidos por quimioterapia alta o moderadamente emetogénica en combinación con un antagonista 5-HT3 + dexametasona (con o sin aprepitant), especialmente después de que la combinación estándar no tenga resultados satisfactorios ${ }^{32}$

32. Un estudio indica que la náusea y el vómito agudos también pueden disminuir con gabapentina. La administración de gabapentina a 300 mg (día 1), 300 mg/dos veces al día (día 2) y 300 mg tres veces al día (días 1 al 5) parece prevenir la náusea y el vómito de inicio tardío en pacientes que recibieron esquemas de quimioterapia de moderada o alta emetogenicidad ${ }^{33}$.

33. Aunque no se encuentra disponible en América Latina, el tratamiento óptimo para prevenir náusea y vómito en pacientes que reciben agentes antineoplásicos altamente emetogénicos es la combinación de antagonista del receptor de neurocinina 1 (días 1 a 3 con aprepipant; solo día 1 con fosaprepipant) + antagonista de serotonina 5-HT3 (día 1 solamente; si es ondasetrón, días 1 y 2) + dexametasona (días 1 a 3 o 1 a 4$)^{13}$. El fosaprepitant ya ha sido aprobado por la EMEA y la FDA ${ }^{30}$.

34. En casos de quimioterapia de dosis alta con trasplante de células progenitores (ya sea de médula ósea o sangre periférica), los pacientes deben recibir una combinación de antagonista del receptor de serotonina 5-HT3 + dexametasona. También, se puede administrar aprepipant, pero la evidencia muestra que su uso es limitado ${ }^{13}$.

35. En la terapia moderadamente emetogénica, se utiliza la combinación de antagonistas de serotonina (5-HT3) + antagonistas de neurocinina + esteroide ${ }^{13}$.

36. En la terapia moderadamente emetogénica, se utiliza la combinación antagonistas de serotonina (5-HT3) (palonosetrón preferentemente) ${ }^{10}$ + antagonistas de neurocinina + esteroide ${ }^{13}$. En general, la combinación de palonosetrón
+ dexametasona logra un mayor control de la náusea y vómito inducidos por quimioterapia que la combinación ondansetrón o granisetrón + dexametasona $a^{10,13,15,25}$.

37. La combinación aprepitant + dexametasona + palonosetrón es efectiva y alcanza respuestas completas generales (ausencia de vómito y de necesidad de medicamentos de rescate) en el $51 \%$ de los pacientes, en el $76 \%$ de estos con náusea y vómito agudo, y en el $66 \%$ de pacientes con CINV de inicio retardado'. Recomendación A-1.

38. Los pacientes tratados con quimioterapia altamente emetogénica deben recibir terapia combinada de antagonista de neurocinina 1 (NK-1) + antagonista 5-HT3 + dexametasona ${ }^{13}$. Recomendación A1.

39. En general, la terapia elegida en casos de quimioterapia emética moderada es palonosetrón + dexametasona ${ }^{13}$.

40. La utilidad de los canabinoides es limitada debido a sus efectos tóxicos: mareo, disforia, alucinaciones $^{10}$.

41. El tratamiento psicológico conductual es apropiado en casos de náusea y vómito anticipatorios, porque reduce los niveles de ansiedad y angustia ${ }^{17}$.

42. No hay terapia complementaria recomendable para el control de la emesis, solo lorazepam en casos de ansiedad extrema ${ }^{13}$.

43. El lorazepam se indica como ansiolítico para reducir la incidencia de náusea y vómito inducidos por quimioterapia anticipatoria, así como en pacientes con emesis refractaria y de aparición súbita ${ }^{10,14,34}$.

44. El lorazepam reduce la incidencia de náusea y vómito anticipatorios; sin embargo, la sedación y amnesia moderadas son comunes ${ }^{16}$.

45. La acupuntura pudiera utilizarse para reducir la náusea y el vómito relacionados con quimioterapia solo cuando el paciente lo solicite, debido a que se carece de evidencia sobre su efectividad ${ }^{17}$.

\section{Conclusiones}

La náusea y el vómito inducidos por fármacos utilizados en quimioterapia son dos de los principales efectos adversos del tratamiento antineoplásico. 
Aunque se ha logrado un avance importante en el manejo y prevención de la náusea y vómito inducidos por quimioterapia en la oncología mundial, mediante la conformación de estas guías, se intenta proveer de recomendaciones -basadas en las guías de MASCC, ASCO, NCCN y de expertos latinoamericanos- a todos los involucrados en la oncología latinoamericana de acuerdo con la disponibilidad de los fármacos en Latinoamérica. De esta forma, se alentará el uso adecuado de los recursos existentes y, con ello, se logrará una mejor calidad en el cuidado de los pacientes oncológicos.

\section{Referencias}

1. Feyer P, Jordan K. Update and new trends in antiemetic therapy: the continuing need for novel therapies. Ann Oncol. 2011;22(1):30-8.

2. Gralla RJ, Osoba D, Kris MG, Kirkbride P, Hesketh PJ, Chinnery LW, et al. Recommendations for the use of antiemetics: evidence-based, clinical practice guidelines. J Clin Oncol. 1999;17(9):2971-94.

3. Chung SK, Ahn MJ, Yoo JY, Choi M, Hyang N, Woo SR, et al. Implementation of best practice for chemotherapy-induced nausea and vomiting in an acute setting. Int J Evid Based Healthc 2011;9(1):32-8.

4. Rodríguez R. Náusea crónica y vómito en el paciente con cáncer. Disponible en: <http://www.paho.org/spanish/ad/dpc/nc/palliative-care-08.pdf>

5. Chambers P, Daniels S. Antiemetic guidelines for adult patients receiving chemotherapy and radiotherapy. University College Hospital NHS Foundation Trust; 2012. p. 1-15.

6. Maule WF. Nausea and vomiting. Chapter 84. In: Walker HK, Hall WD, Hurst JW, editors. Clinical methods: the history, physical, and laboratory examinations. 3rd ed. Boston: Butterworths; 1990.

7. Morrow GR. Chemotherapy-related nausea and vomiting: etiology and management. CA Cancer J Clin. 1989;39(2):89-104.

8. Hesketh PJ. Chemotherapy-induced nausea and vomiting. N Engl J Med. 2008;358(23):2482-94.

9. Herrsted J, Dombernowsky P. Anti-emetic therapy in cancer chemotherapy: current status. Basic Clin Pharmacol Toxicol. 2007; 101(3):143-50.

10. Jordan K, Sippel C, Schmoll HJ. Guidelines for antiemetic treatment for chemotherapy-induced nausea and vomiting: past, present, and future recommendations. The Oncologist. 2007;12(9):1143-50.

11. Valle-Solís AE, Cervantes-Sánchez G, Franco-González EE, García G, Hernández-Chávez GA, López-Hernández $M$, et al. Guías de manejo de antieméticos en oncología, hematología y radioterapia. GAMO. 2011;10 (supl 4):1-20

12. Grunberg SM, Deuson RR, Mavros P, Geling O, Hansen M, Cruciani $G$, et al. Incidence of chemotherapy-induced nausea and emesis after modern antiemetics. Cancer. 2004;100(10):2261-8.

13. Basch E, Prestrud AA, Hesketh PJ, Kris MG, Feyer PC, Somerfield MR, et al. Antiemetics: American Society of Clinical
Oncology clinical practice guideline update. J Clin Oncol. 2011;29(31):4189-98.

14. Roila F, Herrstedt J, Aapro M, Gralla RJ, Einhorn LH, Ballatori E, et al. ASCO guidelines 2011, MASCC y ESMO clinical practice guidelines. Ann Oncol. 2010;21(Suppl 5);v232-43.

15. NCCN clinical practice guidelines in oncology. Antiemesis. 2012. Available in: <http://www.nccn.org/professionals/physician_gls/ pdf/antiemesis.pdf $>$.

16. Roscoe JA, Morrow GR, Aapro MS, Molassiotis A, Olver I. Anticipatory nausea and vomiting. Support Care Cancer. 2011;19(10):1533-8.

17. Aapro MS, Molassiotis A, Olver I. Anticipatory nausea and vomiting. Support Care Cancer. 2005;13(2):117-21.

18. Roila F, Del Favero A, Gralla RJ, Tonato M. Prevention of chemotherapy-and radiotherapy-induced emesis: results of the Perugia Consensus Conference. Ann Oncol. 1998;9(8):811-9.

19. Durand JP, Madelaine I, Scotté F. [Guidelines for prophylaxis and treatment of chemotherapy-induced nausea and vomiting]. Bull Cancer. 2009;96(10):951-60.

20. de Wit R, de Boer AC, vd Linden GH, Stoter G, Sparreboom A, Verweij J. Effective cross-over to granisetron after failure to ondansetron, a randomized double blind study in patients failing ondansetron plus dexamethasone during the first 24 hours following highly emetogenic chemotherapy. $\mathrm{Br} J$ Cancer. 2001;85(8):1099-101.

21. Navari RM. Palonosetron: a second-generation 5-hydroxytryptamine receptor antagonist. Future Oncol 2006;2(5):591-602.

22. Sepúlveda-Vildósola AC, Betanzos-Cabrera Y, Lastiri GG, RiveraMárquez H, Villasis-Keever MA, Del Ángel VW, et al. Palonosetron hydrochloride is an effective and safe option to prevent chemotherapy-induced nausea and vomiting in children. Arch Med Res. 2008;39(6):601-6.

23. Phillips RS, Gopaul S, Gibson F, Houghton E, Craig JV, Light K, et al. Antiemetic medication for prevention and treatment of chemotherapy induced nausea and vomiting in childhood. Cochrane Database Syst Rev. 2010;(9):CD007786.

24. Gore L, Chawla S, Petrilli A, Hemenway M, Schissel D, Chua V, et al. Aprepitant in adolescent patients for prevention of chemotherapy-induced nausea and vomiting: a randomized, double-blind, placebo-controlled study of efficacy and tolerability. Pediatr Blood Cancer. 2009;52(2):242-7.

25. Likun Z, Xiang J, Yi B, Xin D, Liu-Tao Z. A systematic review and meta-analysis of intravenous palonosetron in the prevention of chemotherapy-induced nausea and vomiting in adults. The Oncologist. 2011;16(2):207-16.

26. McCrea JB, Majumdar AK, Goldberg MR, Iwamoto M, Gargano C, Panebianco DL, et al. Effects of neurokinin1 receptor antagonist aprepipant on the pharmacokinetics of dexamethasone and methylprednisolone. Clin Pharmacol Ther. 2003;74(1):17-24.

27. Double-blind, dose-finding study of four intravenous doses of dexamethasone in the prevention of cisplatin-induced acute emesis. Italian Group for Antiemetic Research. J Clin Oncol. 1998;16(9):2937-42.

28. Hesketh PJ, Grunberg SM, Gralla RJ, Warr DG, Roila F, de Wit R, et al. The oral neurokinin-1 antagonist aprepitant for the prevention of chemotherapy-induced nausea and vomiting: a multinational, randomized, double-blind, placebo-controlled trial in patients receiving high-dose cisplatin--the Aprepitant Protocol 052 Study Group. J Clin Oncol. 2003;21(22):4112-9. 
29. Poli-Bigelli S, Rodrigues-Pereira J, Carides AD, Julie Ma G, Eldridge K, Hipple A, et al. Addition of the neurokinin 1 receptor antagonist aprepitant to standard antiemetic therapy improves control of chemotherapy-induced nausea and vomiting. Results from a randomized, double-blind, placebo-controlled trial in Latin America. Cancer. 2003;97(12):3090-8.

30. Grunberg S, Chua D, Maru A, Dinis J, DeVandry S, Boice JA, et al. Single-dose fosaprepitant for the prevention of chemotherapyinduced nausea and vomiting associated with cisplatin therapy: randomized, double-blind study protocol--EASE. J Clin Oncol. 2011;29(11):1495-501.

31. NCCN guidelines version 3.2011. Antiemesis. Available in: $<$ http://www.nccn.org/index.asp >.
32. Cruz FM, de Iracema Gomes Cubero $D$, Taranto $P$, Lerner $T$, Lera AT, da Costa Miranda M, et al. Gabapentin for the prevention of chemotherapy- induced nausea and vomiting: a pilot study. Support Care Cancer. 2012;20(3):601-6

33. Menéndez-Leal $A$, Quijano $C$, Menéndez-Rivera $A$. Is gabapentin effective for preventing delayed NV after moderately and highly emetogenic CT. ASCO 2006 Abstr 18575.

34. Razzavi D, Delvaux N, Farvacques C, De Brier F, Van Heer C, Kaufman $\mathrm{L}$, et al. Prevention of adjustment disorders and anticipatory nausea secondary to adjuvant chemotherapy: a double-blind, placebo-controlled study assessing the usefulness of alprazolam. J Clin Oncol. 1993;11(7):1384-90. 